\title{
Telomerase inhibitor shows promise in myeloproliferative disorders
}

Telomerase inhibitors have been tried as therapies for numerous cancers, including breast cancer and non-small-cell lung cancer, but have not been effective in clinical trials. Two recently published Phase II trials, however, have found that treatment with the telomerase inhibitor imetelstat can lead to clinical responses in patients with essential thrombocythaemia and myelofibrosis (N. Engl. L. Med. 373, 908-919; 2015; N. Engl.J. Med. 373, 920-928; 2015).

Telomerase activity is found in a limited number of cells, notably in cancers and other rapidly dividing tissues, and is needed to maintain telomere length and DNA integrity during cell division, thus providing a rationale for targeting telomerase in patients with hyperproliferative diseases. Telomerase consists of a reverse transcriptase and an RNA template, and imetelstat is a thiophosphoramidate oligonucleotide directed against the RNA component. Other telomerase-targeting strategies, including small molecules and antibodies directed against telomerase, have also been tried as cancer therapies.

Although essential thrombocythaemia (which is characterized by an overproduction of platelets) and myelofibrosis (which is characterized by an overproduction of myeloid cells) are both myeloproliferative disorders, "these two groups of patients are quite different", explains Mary Frances McMullin, a professor of clinical haematology at Queens University Belfast, UK. "Essential thrombocythaemia is a disorder where patients have an [arguably] normal lifespan," she says, whereas for patients with myelofibrosis, "life expectancy is usually a couple of years". Although myelofibrosis can be effectively treated by allogeneic bone marrow transplantation, this therapy is not an option for many patients owing to the associated risks.

In the recent trial of imetelstat in patients with myelofibrosis, $21 \%$ (7 out of 33 ) of patients had a partial or complete response. "The thing that is interesting and exciting is that four people had a complete haematological response and reversal of bone marrow fibrosis and, of those, three had a molecular response," says McMullin. This suggests that imetelstat could be targeting the cause of the disease, rather than just the symptoms. In patients with essential thrombocythaemia, $89 \%$ (16 out of 18 ) of patients had a complete haematological response, and seven of the eight patients assessed had molecular responses.

"I was very positively surprised by the results in these two papers," says Maria Blasco, Director of the Centro Nacional de Investigaciones Oncológicas in Madrid, Spain, "but l'm still very intrigued about the mechanism of action". As noted by the authors, the response to imetelstat was, unexpectedly, independent of telomere length. Telomerase inhibitors are thought to trigger cell death by causing progressive deterioration of telomeres, which triggers a DNA damage response and subsequent apoptosis. In telomerase-deficient mice, "you need to have cells with really short telomeres in order to see the effects of inhibiting telomerase," explains Blasco.

In an accompanying editorial (N. Engl.J. Med. 373, 965-966; 2015), Mary Armanios and Carol Greider, both from Johns Hopkins University School of Medicine, Baltimore, USA, suggest that imetelstat could be working not through inhibition of telomerase, but instead through effects that are common to multiple phosphorothioate antisense oligonucleotides. They argue that myelosuppression is commonly observed with this class of therapeutics; indeed, their argument is strengthened by the observed side effects of this drug, which include grade 3 and 4 neutropaenia, thrombocytopaenia and anaemia. "Normal tissue has much longer telomeres than cancers - particularly the stem cell compartment, which has the longest telomeres in the body - so I would have expected a bigger therapeutic window [if the compounds were working though telomerase inhibition]," agrees Blasco.

Regardless of the mechanism of action, imetelstat seems to be effective in patients with myeloproliferative disorders, who currently have limited therapeutic options. "Imetelstat gives molecular responses, which is very exciting, but the toxicity is going to be an issue," says McMullin. Further ongoing clinical studies could clarify the mechanism of action and identify strategies to minimize toxicity. Megan Cully
I'm still very

intrigued

about the

mechanism

of action

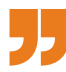

